\title{
SAMHD1 restricts HIV-1 reverse transcription in quiescent $\mathrm{CD} 4^{+} \mathrm{T}$-cells
}

Benjamin Descours ${ }^{1 *}$, Alexandra Cribier ${ }^{1}$, Christine Chable-Bessia ${ }^{1}$, Diana Ayinde ${ }^{3}$, Gillian Rice ${ }^{2}$, Yanick Crow ${ }^{2}$, Ahmad Yatim ${ }^{1}$ Olivier Schwartz ${ }^{3}$, Nadine Laguette ${ }^{1}$ and Monsef Benkirane ${ }^{1 *}$

\begin{abstract}
Background: Quiescent CD4 ${ }^{+}$T lymphocytes are highly refractory to HIV-1 infection due to a block at reverse transcription.

Results: Examination of SAMHD1 expression in peripheral blood lymphocytes shows that SAMHD1 is expressed in both CD4+ and CD8+ T cells at levels comparable to those found in myeloid cells. Treatment of CD4+ T cells with Virus-Like Particles (VLP) containing Vpx results in the loss of SAMHD1 expression that correlates with an increased permissiveness to HIV-1 infection and accumulation of reverse transcribed viral DNA without promoting transcription from the viral LTR. Importantly, CD4 ${ }^{+}$T-cells from patients with Aicardi-Goutières Syndrome harboring mutation in the SAMHD1 gene display an increased susceptibility to HIV-1 infection that is not further enhanced by VLP-Vpx-treatment.

Conclusion: Here, we identified SAMHD1 as the restriction factor preventing efficient viral DNA synthesis in non-cycling resting $\mathrm{CD}^{+}$T-cells. These results highlight the crucial role of SAMHD1 in mediating restriction of HIV-1 infection in quiescent CD4 ${ }^{+}$T-cells and could impact our understanding of HIV-1 mediated CD4 ${ }^{+}$T-cell depletion and establishment of the viral reservoir, two of the HIV/AIDS hallmarks.
\end{abstract}

Keywords: SAMHD1, Quiescent CD4 ${ }^{+}$T-cell, HIV-1, Reverse transcription, Restriction

\section{Background}

The human immunodeficiency virus type-1 (HIV-1) primarily infects $\mathrm{CD}^{+} \mathrm{T}$-cells. While activated lymphocytes support viral replication, non-cycling quiescent $\mathrm{CD}_{4}^{+} \mathrm{T}$ cells allow entry of HIV-1 but fail to allow efficient and complete reverse transcription [1-6]. However, addition of deoxynucleosides to unstimulated lymphocytes cultures partially overcomes this failure [7-9], suggesting that an insufficient supply of deoxynucleotide triphosphates (dNTPs) in quiescent T-cells may, at least in part, contribute to inefficient viral DNA synthesis $[8,10]$. Interestingly, the Aicardi-Goutières syndrome gene product SAMHD1 was recently described as the restriction factor that blocks HIV-1 infection of non-cycling myeloid cells [11-13]. SAMHD1 is a dGTP-dependent deoxynucleotide triphosphohydrolase [14-16] that reduces the cellular pool of dNTPs in differentiated, non-cycling myeloid cells to levels

\footnotetext{
*Correspondence: Benjamin.descours@igh.cnrs.fr; bmonsef@igh.cnrs.fr ${ }^{1}$ Institut de Génétique Humaine, CNRS UPR1142, Laboratoires de Virologie Moléculaire, Montpellier, France

Full list of author information is available at the end of the article
}

below those required to support HIV-1 DNA synthesis $[15,17]$. However, SAMHD1's spectrum of activity beyond cells of the myeloid lineage remains unclear.

\section{Results and discussion}

We first determined whether SAMHD1 was expressed in peripheral blood lymphocytes from healthy donors. Western blot analysis revealed that both $\mathrm{CD}_{4}^{+}$and $\mathrm{CD}^{+} \mathrm{T}$ cells express SAMHD1 (Figure 1a). The expression levels of SAMHD1 in unstimulated $\mathrm{CD} 4^{+} \mathrm{T}$-cells were similar to those found in myeloid cells, including $\mathrm{CD} 14^{+}$monocytes, monocytes-derived macrophages (MDM) and monocytederived dendritic cells (MDDC) (Figure 1a). Moreover, flow cytometry analysis revealed that all circulating $\mathrm{CD} 4^{+}$ T-cells subsets, including naïve ( $\mathrm{Tn} ; \mathrm{CD} 45 \mathrm{RA}^{+}, \mathrm{CCR} 7^{+}$), central memory (Tcm; CD45RA $\left.{ }^{-}, \mathrm{CCR}^{+}\right)$and effector memory cells (Tem; CD45RA', CCR7 ${ }^{-}$), expressed high levels of SAMHD1 (Figure 1b), raising the possibility that SAMHD1 could affect the susceptibility of resting $\mathrm{CD} 4^{+}$ lymphocytes to HIV-1 infection.

\section{Biomed Central}


a

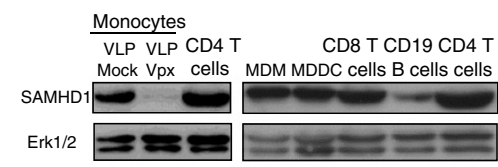

C

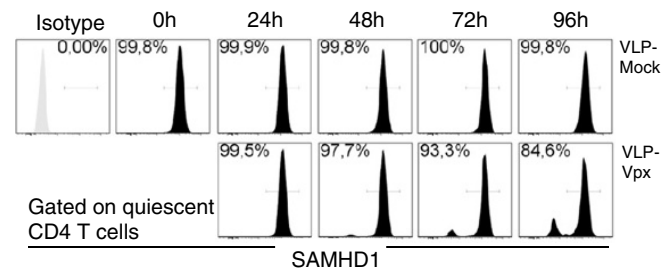

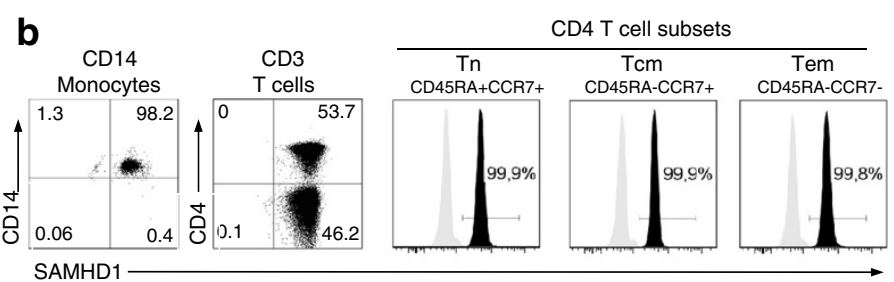

d

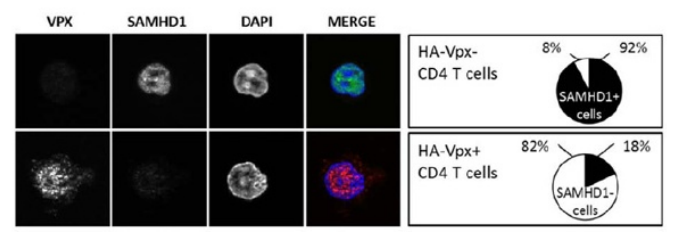

Figure 1 CD4 T lymphocytes express SAMHD1. a. Western blot analysis of SAMHD1 expression in primary hematopoietic cells. $\mathbf{b}$. Flow cytometry analysis of SAMHD1 expression levels in CD14 $4^{+}$monocytes, T-cells (CD4 ${ }^{+}$and CD8 ${ }^{+}$T lymphocytes) and in CD4 ${ }^{+}$T-cell subsets

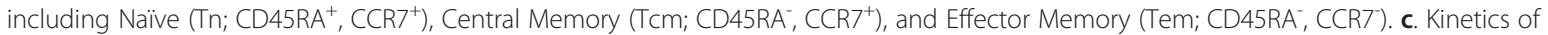
SAMHD1 loss in quiescent (CD69-, HLA-DR') CD4 ${ }^{+}$T-cells after VLP-Vpx treatment (representative experiment, $\left.n=2\right)$. d. Vpx transduction of activated $C D 4^{+}$T-cells induces loss of SAMHD1. TCR-stimulated CD4 ${ }^{+}$T-cells were transduced with Vpx-HA expressing retroviral construct. Fortyeight hours later, SAMHD1 and Vpx were immunostained using specific antibodies. Nuclei were stained in mounting media with DAPI. Pie charts represent the proportions of SAMHD1 ${ }^{+}$cells among $100 \mathrm{Vpx}$ positive and negative counted cells.

To test this hypothesis, we exposed unstimulated peripheral blood mononuclear cells (PBMCs) to viral-like particles containing the SIV accessory protein $\mathrm{Vpx}_{\mathrm{mac} 251}$ (VLP-Vpx), which counteracts SAMHD1-mediated restriction by triggering its proteasomal degradation $[12,13]$. PBMCs treated with VLP-Vpx or empty VLPs (VLP-Mock) were analyzed for SAMHD1 expression by flow cytometry. An average of 16\% of VLP-Vpx treated resting $\mathrm{CD}^{+}{ }^{+}$-cells were SAMHD1 negative at 96 hours post-exposure (Figure 1c), whereas most monocytes (90.2\%) lost the expression of SAMHD1 after $48 \mathrm{hrs}$ of VLP-Vpx treatment (Additional file 1: Figure S1). The difference in Vpx-mediated loss of SAMHD1 in quiescent $\mathrm{CD} 4^{+} \mathrm{T}$-cells and monocytes might result from discrepancies in nuclear/cytoplasmic exchange [18]. Indeed, we have recently shown that $\mathrm{Vpx}$-mediated degradation of SAMHD1 requires its nuclear export $[19,20]$. In support of this hypothesis, loss of SAMHD1 expression was observed in more than $80 \%$ of T-cell receptor (TCR)-stimulated $\mathrm{CD}^{+}$lymphocytes expressing Vpx (Figure 1d). We next evaluated the effect of SAMHD1 loss on HIV1-resistance phenotype of resting $\mathrm{CD} 4^{+}$T-cells. Restriction of HIV-1 replication in quiescent T-cells has been attributed to blocks at both the reverse transcription step and viral gene expression (e.g.: lack of transcription factors such as NF-kB and CyclinT required for viral transcription) $[21,22]$. Thus, to bypass the transcriptional block, we performed single-round infection experiments using an HIV-1 based lentiviral vector carrying an EGFP cassette under the transcriptional control of the CMV promoter (HIV-CMV-EGFP). Unstimulated PBMCs isolated from healthy donors were exposed for $12 \mathrm{hrs}$ to VLP-Vpx or VLP-Mock and subsequently infected with HIV-CMV-EGFP. As expected, no EGFP was detected when cells were treated with VLP-Mock (Figure 2a), while, VLP-Vpx treatment resulted in EGFP expression in quiescent (HLA-DR ${ }^{-}, \mathrm{CD}^{-} 9^{-}$) $\mathrm{CD}^{+}{ }^{+}$T-cells (Figure 2a). An average of $14 \%$ of VLP-Vpx treated resting $\mathrm{CD}^{+} \mathrm{T}$ lymphocytes expressed EGFP, while less than $1 \%$ of cells exposed to VLP-Mock were EGFP+ (Figure 2a and Additional file 1: Figure S2a). As a control, VLP-Vpx treatment enhanced the permissiveness of $\mathrm{CD}_{14}{ }^{+}$monocytes to HIV-CMV-EGFP (Additional file 1: Figure S3b). These results indicate that $\mathrm{Vpx}$ could alleviate the post-entry block to HIV-1 infection of unstimulated T-cells. Importantly, VLP-Vpx treatment was not associated with CD4 ${ }^{+}$ T-cell activation (Additional file 1: Figure S2c) or proliferation (Figure 2b). Vpx increased the susceptibility of non-cycling quiescent T-cells (eFluor ${ }^{\text {High }}$ ) (Figure 2b) while it had no effect on the permissiveness of activated dividing T-cells (eFluor ${ }^{\text {Low }}$ ) despite high level expression of SAMHD1 (Additional file 1: Figure S2d). In addition, Vpx increased HIV-1 infection of all resting $\mathrm{CD}^{+}{ }^{+} \mathrm{T}$ subsets, including highly refractory naïve cells (Tn) (Figure 2c).

We then focused our attention on viral reverse transcription, which is initiated in most HIV-1 exposed Tcell subsets [6]. Completion of this step is, nonetheless, reached in resting CD4+ T-cells at a much slower rate [1-6]. We asked whether SAMHD1 is responsible for the 

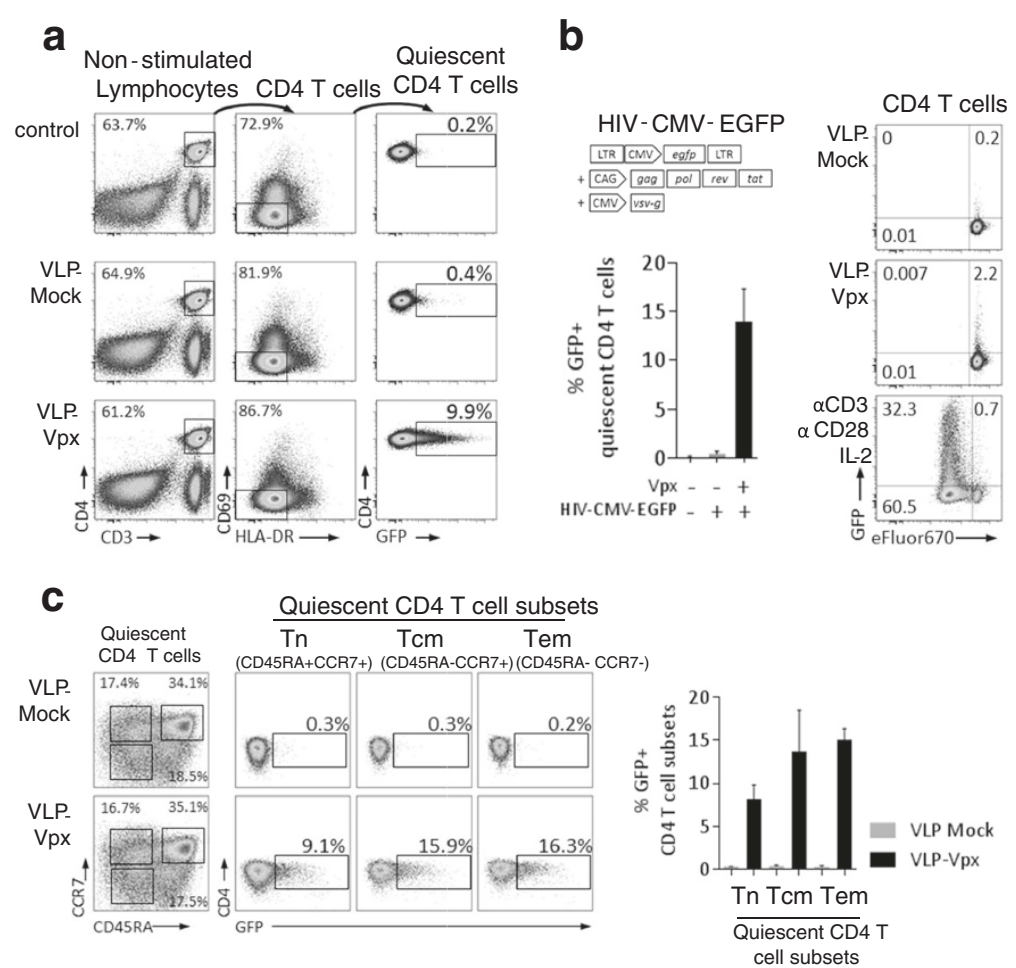

d

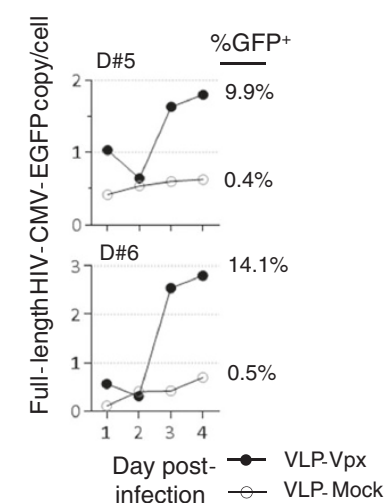

e

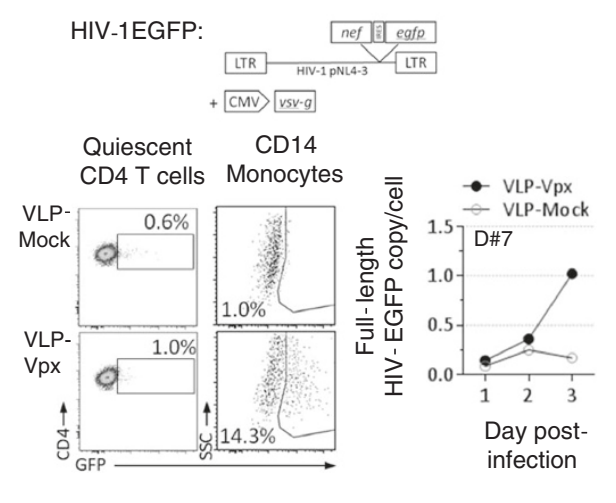

Figure 2 Vpx treatment of quiescent $\mathrm{CD}^{+}{ }^{+}$T-cells results in increased susceptibility to HIV-1 infection. a. Impact of Vpx treatment on quiescent CD4 $4^{+}$T-cells susceptibility to HIV-CMV-EGFP infection. Upper right panel: expression vectors used to produce HIV-CMV-EGFP virions. Left panel: PBMCs were treated $12 \mathrm{~h}$ with VLP-Mock or VLP-Vpx then infected with HIV-CMV-EGFP or uninfected (control). Proportions of EGFP positive quiescent CD4 $4^{+}$T-cells (CD69- HLA-DR-) were assessed 4 days post infection. Lower right panel: Results are expressed as percentage of EGFP positive cells. Graphic shows mean and standard deviation for 6 healthy donors. b. PBMCs were cultured as in $\mathbf{a}$. after staining with the eFluor670 proliferation dye. Cell division and EGFP expression were assessed in CD4 ${ }^{+} \mathrm{T}$-cells (a representative experiment is shown). c. Impact of Vpx on quiescent $C D 4^{+}$T-cell subsets susceptibility to HIV-CMV-EGFP infection. Left panel: gating strategy to define CD4 ${ }^{+}$T-cell subsets: Naïve (Tn; $\mathrm{CD} 4 \mathrm{RA}^{+}, \mathrm{CCR7}^{+}$), Central Memory (Tcm; CD45RA- $\mathrm{CCR}^{+}$) and Effector Memory (Tem; CD45RA- CCR7). Middle panel: Permissiveness of Tn, Tcm and Tem to HIV-CMV-EGFP performed as described in a (a representative experiment; $n=3$ ). $\mathbf{d}$. Kinetics of full length HIV-CMV-EGFP DNA accumulation quantified by quantitative PCR in purified non-stimulated CD4 ${ }^{+}$T-cells from 2 healthy donors \#5 and \#6. Proportions of EGFP positive quiescent CD4 $4^{+}$T-cells (CD69- HLA-DR-) assessed by flow cytometry 4 days post infection by HIV-CMV-EGFP are indicated on the right. e. Effect of Vpx on the permissiveness of quiescent CD4 ${ }^{+}$T-cells to infection by HIV-EGFP. Upper panel: expression vectors used to produce HIV-EGFP virions. Lower left panel: PBMCs were cultured as in a. except cells were infected with HIV-EGFP. Proportions of EGFP positive quiescent CD4 ${ }^{+}$T-cells (CD69- HLA-DR-) and CD14 $4^{+}$monocytes were assessed 4 days post infection for 3 donors. Lower right panel: Full length HIV-EGFP DNA accumulation was performed as in $\mathbf{d}$. for 1 donor. 
efficient reverse transcription block in resting T-cells, as it has been demonstrated for myeloid cells $[13,17]$. PBMCs were exposed to VLP-Vpx or VLP-Mock and infected with HIV-CMV-EGFP. The kinetics of reverse transcription leading to production of full-length HIV-1 DNA in unstimulated CD4+ T-cells was determined by quantitative PCR. We observed that both the amount and the rate of reverse transcription leading to the production of full length viral DNA were enhanced in Vpx treated resting CD4+ T-cells compared to VLP-Mock treated counterparts (Figure 2d). These results show that VLP-Vpx overcomes the restriction of HIV-CMV-EGFP infection in resting $\mathrm{CD}^{+}{ }^{+} \mathrm{T}$-cells by promoting the accumulation of full length reverse transcripts. We next verified whether the observed effect of Vpx applies to wild type HIV-1. For this purpose, unstimulated PBMCs were treated with VLP-Mock or with VLP-Vpx and subsequently infected with HIV-1 expressing EGFP (HIV-EGFP) (Figure 2e). Following infection, we did not detect significant EGFP expression in both VLP-Mock- and VLP-Vpx-treated resting $\mathrm{CD}^{+}{ }^{+} \mathrm{T}$-cells (Figure 2e and Additional file 1: Figure S3a). This is consistent with the HIV-1 LTR transcriptional block associated with their quiescent status and confirms that the analyzed CD4+ T-cells are indeed in a resting state. As a control, VLP-Vpx enhanced the permissiveness to HIV-EGFP of $\mathrm{CD}^{+} 4^{+}$monocyte population (Figure 2e and Additional file 1: Figure S3a). A potential infectivity defect was ruled out, since TCR-mediated activation of T-cells efficiently induced EGFP expression (Additional file 1: Figure S3b). Interestingly, while no EGFP positive cells were detected in resting CD4+ T-cells, an accumulation of HIV-1 full length DNA was observed in VLP-Vpx treated cells (Figure 2e). Thus, Vpx promotes the accumulation of full-length viral DNA following the infection of resting CD4+ T-cells, but does not relieve the transcriptional block required for viral gene expression. The ability of $\mathrm{Vpx}$ to promote infection was further confirmed in another model of resting lymphocytes (Additional file 1: Figure S4). Purified CD4+ T cells were activated with PHA and cultured in IL-2 for 14-20 days, until disappearance of the CD69 and Ki67 activation markers [23]. Treatment of such cells with VLP-Vpx induced SAMHD1 loss in a large fraction of the cells, and a 6-fold increase in their sensitivity to HIV-CMVGFP infection (Additional file 1: Figure S4). Importantly, the majority of infected EGFP-positive cells were found in the SAMHD1-negative cell subset (Additional file 1: Figure S4). Taken together, these results indicate that Vpx, acting through SAMHD1, facilitates infection of resting CD4+ T-cells by promoting the accumulation of fully reverse transcribed viral DNA in quiescent lymphocytes.

To confirm the role of SAMHD1 in the ability of Vpx to overcome HIV-1 restriction in quiescent CD4+ T-cells, we used PBMCs isolated from 4 Aicardi-Goutières syndrome patients harboring homozygous inactivating mutations in the $S A M H D 1$ gene (AGS-5, referred to as $S A M H D 1^{-1-}$ ) [24]. Heterozygous donors for these $S A M H D 1$ mutations (referred to as $S A M H D 1^{-1+}$ ) were used as controls. We first assessed the intrinsic susceptibility of unstimulated $S A M H D 1^{-1-}$ and SAMHD1 $1^{-1+}$ CD4+ T-cells to HIV-CMVEGFP infection. While heterozygous deletion of SAMHD1 did not affect the intrinsic resistance of unstimulated PBMCs to HIV-CMV-EGFP infection, homozygous deletion increased the susceptibility of both quiescent CD4+ T-cells and monocytes (Figure $3 \mathrm{a}, \mathrm{b}, \mathrm{c}$ ), indicating that SAMHD1 is required to mediate HIV-1 restriction in resting CD4+ T-cells. Remarkably, VLP-Vpx treatment did not further enhance permissiveness of $S A M H D 1^{-1-}$ resting CD4+ T-cells (Figure 3b, d). However, the restrictive phenotype of $S A M H D 1^{-/+}$cells is alleviated after VLP-Vpx delivery (Figure $3 \mathrm{a}, \mathrm{d}$ ), indicating that SAMHD1 is required for $\mathrm{Vpx}$ to overcome HIV-1 restriction in T-cells. Overall, these results demonstrate that SAMHD1 acts as an effective HIV-1 restriction factor in non-cycling resting $\mathrm{CD} 4^{+}$lymphocytes.

\section{Conclusion}

The demonstration that SAMHD1 restricts HIV-1 replication in quiescent CD4+ T-cells could have an important implication in our understanding of HIV-1-mediated CD4+ T-cell depletion and establishment of the viral reservoir, two of the HIV/AIDS hallmarks. It has recently been shown that abortive HIV-1 reverse transcription in resting CD4+ T-cells leads to the accumulation of cytoplasmic viral nucleic acids that trigger a host defense program eliciting a coordinated proapoptotic and proinflammatory response $[25,26]$. By preventing completion of reverse transcription in quiescent $\mathrm{CD}_{4}^{+}$T-cells, SAMHD1 could contribute to their depletion. The HIV1 reservoir, which mainly consists of quiescent $\mathrm{CD}^{+} \mathrm{T}-$ cells that harbor integrated silent provirus, represents a major barrier to viral eradication by antiretroviral therapy. It has recently been shown that chemokines can facilitate early steps of HIV-1 replication in resting $\mathrm{CD}_{4}^{+} \mathrm{T}$-cells, leading to latency [27]. It will be of importance to determine whether chemokines regulate SAMHD1 activity and facilitate the generation of latently infected cells in vivo. The regulation of SAMHD1 activity remains an important area of study. In this regard, we observed that SAMHD1 restriction activity does not correlate with its expression levels. Indeed, although SAMHD1 expression is independent of the activation state of $\mathrm{CD}^{+}{ }^{+} \mathrm{T}$-cell, its restriction activity is witnessed only when the cells are in a quiescent state (Additional file 1: Figure S2a and S2d). SAMHD1 activity could be regulated through post-translational modifications and/or through the expression of a cellular partner in non-cycling cells, including quiescent 


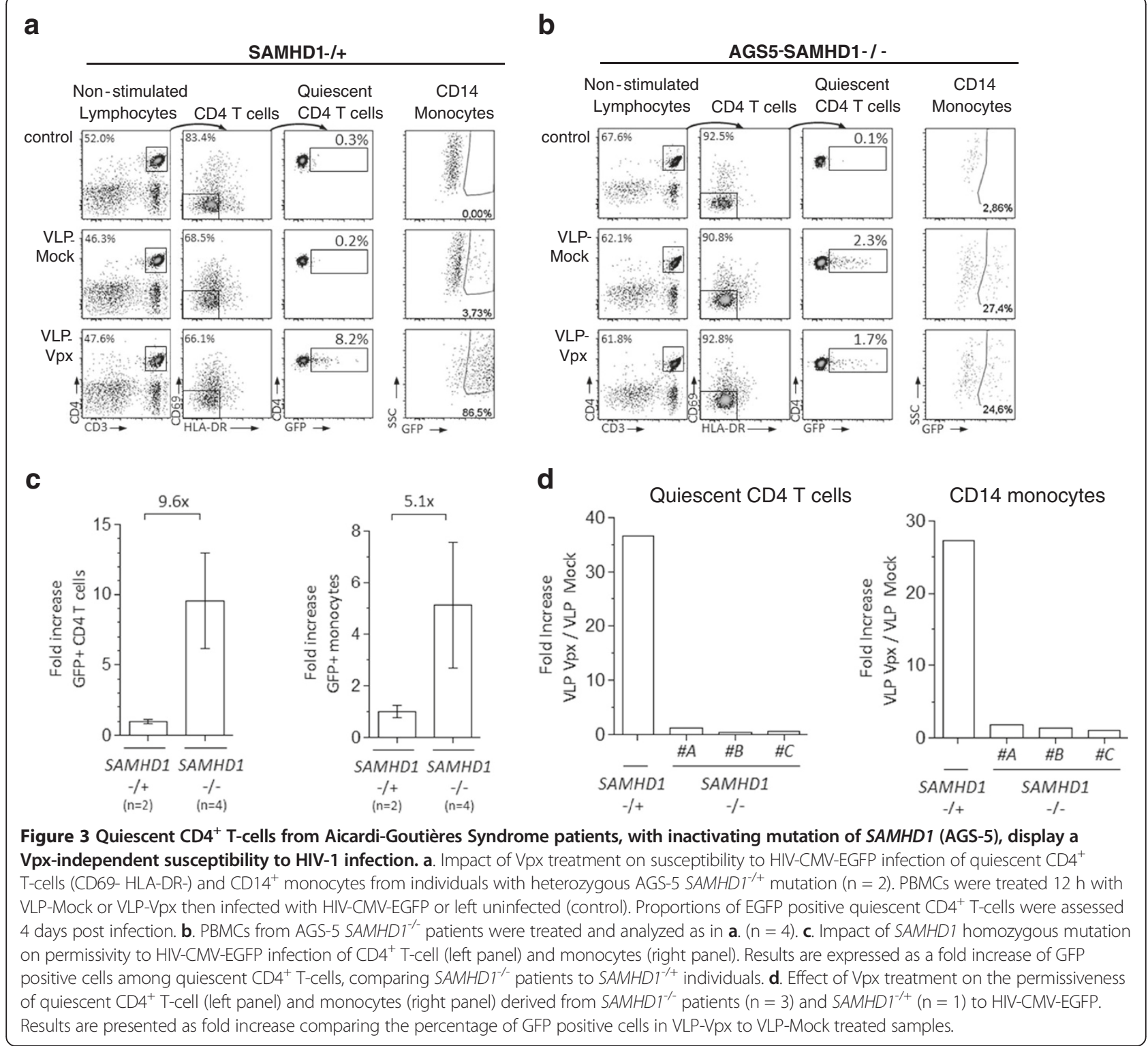

CD4 ${ }^{+}$T-cells. Additionally, SAMHD1 activity can also be regulated through the expression of splice variants lacking the enzymatic activity [28]. Given that dN supply only partially rescues HIV-1 reverse transcription in resting CD4+ T-cells, one can ask whether the restriction imposed by SAMHD1 is fully or partially due to its dNTP triphosphohydrolase activity. Interestingly, it has recently been shown that SAMHD1 is a nucleic acid binding protein that displays a preference for RNA over DNA [29]. Further studies are required to elucidate the mechanism by which SAMHD1 restricts HIV-1 in resting CD4+ T-cells. Deciphering the functional interaction between HIV-1 and SAMHD1 will lead to a better understanding of the damage imposed by this virus to the immune system and the progression towards AIDS.

\section{Methods}

\section{Cell extract and western blot analysis} microbeads or $\mathrm{CD} 4^{+}$T-cell isolation kit (Miltenyi Biotec). CD14 ${ }^{+}$monocytes were treated 2 hours with VLPVpx before preparation of whole cell extracts (WCE). WCE were prepared with buffer containing $0.5 \%$ Triton $\mathrm{X}-100,150 \mathrm{mM} \mathrm{NaCl}, 10 \mathrm{mM} \mathrm{KCL}, 1.5 \mathrm{mM} \mathrm{MgCl}$, $0.5 \mathrm{mM}$ EDTA $10 \mathrm{mM} \beta$-mercaptoethanol, $0.5 \mathrm{mM}$ PMSF. Cell lysates were boiled in SDS sample buffer and resolved on a SDS-PAGE gel (Biorad). Proteins were liquid-transferred (Biorad) to nitrocellulose membrane in transfer buffer (20\% methanol, $25 \mathrm{mM}$ Tris, $192 \mathrm{mM}$ Glycine, 0.037\% SDS) during $90 \mathrm{~min}$ at $100 \mathrm{~V}$. Western blotting was performed using the following antibodies:
$\mathrm{CD}_{1}{ }^{+}$and $\mathrm{CD} 4^{+}$T-cells were purified using $\mathrm{CD} 14^{+}$ 
mouse anti-SAMHD1 (Abcam \#AB67821) and rabbit anti-Erk1/2 (Cell Signaling Technology).

\section{Immunofluorescence}

Purified $\mathrm{CD}_{4}^{+} \mathrm{T}$-cells were stimulated for 3 days with anti-CD3, anti-CD28 and IL-2 and transduced with Vpx expressing retroviral construct [13]. Two days post transduction, cells were harvested and fixed in PBS with $4 \%$ paraformaldehyde and $2 \%$ sucrose, and permeabilized with $0.5 \%$ Triton X-100, $20 \mathrm{mM}$ Tris (pH 7.6), $50 \mathrm{mM}$ $\mathrm{Nacl}, 3 \mathrm{mM} \mathrm{MgCl} 2$, and $300 \mathrm{mM}$ sucrose. Wash and antibody incubation steps were performed in PBS-0.1\% Tween. Cells were stained with Anti-SAMHD1 (Abcam \#AB67821) and Vpx was stained with anti-HA (Covance). Secondary antibodies were purchased from Invitrogen. Nuclei were stained with DAPI in mounting media (Vectashield; Vector Labs) and images were collected on a Zeiss Axioimager Apotome.

\section{Plasmids}

$\mathrm{SIV}^{+}$was kindly provided by N. Manel. SIV- was a gift from J. Luban. HA-Vpx $\mathrm{mac}_{251}$ was subcloned in $\mathrm{pOz-}$ IL2R $\alpha$ expression vector. pHRET was kindly provided by C. Mettling. pBR-NL4-3-IRES-EGFP was a gift of F. Kirchhoff. psPAX2 packaging plasmid was obtained from Addgene (D. Trono). MMLV packaging plasmid, A-MLV envelope and pMD2-G VSV-G envelope were previously described [13].

\section{Virus-Like Particles (VLP) and virus production}

VLPs and viral particles were produced from $293 \mathrm{~T}$-cells using the standard phosphate calcium transfection protocol. For VLPs, 293 T-cells were transfected with $8 \mu \mathrm{g} \mathrm{SIV}{ }^{+}$and $2 \mu \mathrm{g}$ pMD2-G VSV-G encoding plasmid (VLP-Vpx) or with $8 \mu \mathrm{g}$ SIV- and $2 \mu \mathrm{g}$ pMD2-G (VLPMock). Media were replaced $16 \mathrm{~h}$ after transfection, and VLPs were harvested $48 \mathrm{~h}$ later, filtered at $0.45 \mu \mathrm{m}$ and concentrated 100 times by ultracentrifugation. For virus production, HIV-EGFP was produced by transfection of $10 \mu \mathrm{g}$ of pBR-NL4-3-IRES-EGFP and $1 \mu \mathrm{g}$ of pMD2-G. HIV-CMV-EGFP was produced by transfection of $5 \mu \mathrm{g}$ of pHRET, $5 \mu \mathrm{g}$ of psPAX2 packaging vector, and $2.5 \mu \mathrm{g}$ of pMD2-G. For MLV transduction particles, 293 T-cells

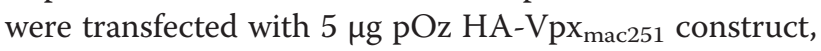
$2.5 \mu \mathrm{g}$ MMLV packaging plasmid, and $2.5 \mu \mathrm{g}$ A-MLV envelope encoding plasmid. When required, p24 concentration was measured by ELISA (Innogenetics).

\section{Infection}

PBMCs were isolated from blood samples by Ficoll gradient (Eurobio), cultured $12 \mathrm{~h}$ in presence of VLP$\mathrm{Vpx}_{\text {mac251 }}$ or VLP-Mock at a density of 2 million cells per well (24 well plates) in $300 \mu \mathrm{l}$ of $10 \%$ FCS supplemented complete RPMI (R10) (Invitrogen). Infection was then performed by addition of $300 \mu$ of R10 diluted HIV-CMV-EGFP $(1 \mu \mathrm{g})$ or HIV-EGFP (800 ng) for 4 days before analysis. As control, TCR stimulated PBMCs were cultured for 3 days on plate-bound anti-CD3 antibody $(5 \mu \mathrm{g} / \mathrm{ml})$ (Miltenyi Biotec) in presence of $1 \mu \mathrm{g} / \mathrm{ml}$ antiCD28 antibody and IL-2 (20U/ml) (Roche) and infected with HIV-CMV-EGFP $(1 \mu \mathrm{g})$ or HIV-EGFP $(800 \mathrm{ng})$ for 2 days before analysis. Optimal viral inoculums were determined by titration using TCR stimulated PBMCs. Alternatively, after thawing, Trypan Blue viability assessment and TURBO ${ }^{\mathrm{TM}}$ DNase (Ambion) treatment at $37^{\circ} \mathrm{C}$ for 1 hour were performed, and cryopreserved PBMCs were cultured in aforementioned conditions.

\section{Flow cytometry}

Non-cycling, quiescent $\mathrm{CD} 4^{+} \mathrm{T}$-cells and monocytes were analyzed using the following antibodies and dye: BrilliantViolet421 anti-CD3 (UCHT1), PeCy7 anti-CD4 (RPA-T4), PE anti-CD69 (FN50), APC anti-HLA-DR (TU36), V500 anti-CD14 (M5ED), PeCy7 anti-CCR7 (3D12), PE antiCD45RA (HI100) (BD Biosciences), PerCP anti-CD45 (5B1) (Miltenyi Biotec), Cell-Proliferation Dye eFluor ${ }^{\circledR}$ 670 (eBiosciences) and Alexa-488 Anti-SAMHD1 (I-1918) (O. Schwartz). Cells were analyzed on MACSQuant Analyzer (Milteny Biotec). Data analyses were performed on FlowJo (TreeStar inc).

\section{Quantification of viral full-length DNA}

Prior infection, viral stocks were treated for $1 \mathrm{~h}$ at $37^{\circ} \mathrm{C}$ with $100 \mathrm{U} / \mathrm{ml}$ of TURBO ${ }^{\mathrm{TM}}$ DNase (Ambion). PBMCs (2 X $10^{6}$ cells) were infected with $1000 \mathrm{ng}$ of HIV-CMVEGFP or with heat inactivated HIV-CMV-EGFP. CD4 ${ }^{+}$ T-cells were purified from cultured PBMCs by depleting non $\mathrm{CD}_{4}^{+} \mathrm{T}$-cell, using $\mathrm{CD} 4^{+} \mathrm{T}$-cell isolation kit (Miltenyi Biotec) (Average purification yield of 97\%). Genomic DNA was extracted using the QIAmp DNA blood minikit (Qiagen). Full-length viral DNA quantification was performed by quantitative PCR using primers annealing in the 5'LTR-U5 and gag regions. PCR measurements were performed in duplicate using SYBR Green (Qiagen). Amplifications were carried out in the LightCycler480 (Roche). The average of the technical duplicates was normalized to GAPDH levels using the comparative CT method $(2 \Delta \Delta \mathrm{CT})$.

\section{Preparation of resting post-activated CD4+ T cells and lentiviral vector transduction}

$\mathrm{CD} 4+\mathrm{T}$ cells were isolated by positive selection as described above (Miltenyi Biotec). Resting CD4+ T cells were activated with $1 \mathrm{ug} / \mathrm{ml}$ phytohemagglutinin (PHA) and $100 \mathrm{U} / \mathrm{ml}$ Interleukin 2 (IL-2) and cultured in fresh medium containing IL-2 for 14 to 20 days [23]. The activation state was monitored every few days by flow cytometry after staining with PE-coupled CD69 and 
FITC-coupled Ki67 (BD Pharmingen). Resting postactivated cells were treated with Vpx-VLPs or MockVLPs for 3 hours, washed and incubated overnight with HIV-CMV-EGFP. The following day, cells were washed and incubated in fresh medium containing IL-2 for 96 hours. Percentages of EGFP positive and SAMHD1 negative cells were then assessed by flow cytometry.

\section{Additional file}

Additional file 1: SAMHD1 restricts HIV-1 replication in quiescent CD4+ T-cells.

\section{Competing interests}

The authors declare that they have no competing interests.

\section{Authors' contributions}

$\mathrm{BD}$ performed most of the experiments. $\mathrm{AC}$ and $\mathrm{CCB}$, performed some experiments. OS and DA designed and performed the experiment shown in Additional file 1: figure S4. GR and YC provided AGS5 cells. BD and MB designed the experiments and wrote the manuscript. All authors read and approved the final manuscript.

\section{Acknowledgments}

We thank Rosemary Kiernan and members of the Molecular Virology Lab for critical reading of the manuscript. We thank the Montpellier RIO imaging facility (MRI) for providing adequate environment for microscopy experiments. We thank Clément Mettling for providing us pHRET for HIVCMV-EGFP production. This work was supported by grants from the ERC (250333), Sidaction (fonds de dotation Pierre Bergé), ANRS and FRM "équipe labéllisée" to MB. BD was supported by ERC fellowships; AC by ANRS, NL by SIDACTION and YA by MESR, Ecole de I'INSERM-Liliane Bettencourt and FRM fellowships. Work in OS lab was supported by ANRS, Sidaction, Areva, the Labex IBEID program, Areva, and the Vaccine Research Institute. DA was supported by ANRS.

\section{Author details}

'Institut de Génétique Humaine, CNRS UPR1142, Laboratoires de Virologie Moléculaire, Montpellier, France. ${ }^{2}$ Academic Unit of Medical Genetic, University of Manchester, Manchester, UK. ${ }^{3}$ Institut Pasteur, Virus and Immunity Unit, URA CNRS 3015, Paris, France.

Received: 5 October 2012 Accepted: 16 October 2012

Published: 23 October 2012

\section{References}

1. Pierson TC, Zhou Y, Kieffer TL, Ruff CT, Buck C, Siliciano RF: Molecular characterization of preintegration latency in human immunodeficiency virus type 1 infection. J Virol 2002, 76:8518-8531.

2. Spina CA, Guatelli JC, Richman DD: Establishment of a stable, inducible form of human immunodeficiency virus type 1 DNA in quiescent CD4 lymphocytes in vitro. J Virol 1995, 69:2977-2988.

3. Stevenson M, Stanwick TL, Dempsey MP, Lamonica CA: HIV-1 replication is controlled at the level of $\mathrm{T}$ cell activation and proviral integration. EMBO J 1990, 9:1551-1560.

4. Swiggard WJ, O'Doherty U, McGain D, Jeyakumar D, Malim MH: Long HIV type 1 reverse transcripts can accumulate stably within resting CD4+ T cells while short ones are degraded. AIDS Res Hum Retroviruses 2004, 20:285-295

5. Vatakis DN, Bristol G, Wilkinson TA, Chow SA, Zack JA: Immediate activation fails to rescue efficient human immunodeficiency virus replication in quiescent CD4+ T cells. J Virol 2007, 81:3574-3582.

6. Zack JA, Arrigo SJ, Weitsman SR, Go AS, Haislip A, Chen IS: HIV-1 entry into quiescent primary lymphocytes: molecular analysis reveals a labile, latent viral structure. Cell 1990, 61:213-222.

7. Dai J, Agosto LM, Baytop C, Yu JJ, Pace MJ, Liszewski MK, O'Doherty U: Human immunodeficiency virus integrates directly into naive resting
CD4+ T cells but enters naive cells less efficiently than memory cells. J Virol 2009, 83:4528-4537.

8. Korin YD, Zack JA: Nonproductive human immunodeficiency virus type 1 infection in nucleoside-treated G0 lymphocytes. J Virol 1999, 73:6526-6532.

9. Plesa G, Dai J, Baytop C, Riley JL, June CH, O'Doherty U: Addition of deoxynucleosides enhances human immunodeficiency virus type 1 integration and 2LTR formation in resting CD4+ T cells. J Virol 2007, 81:13938-13942.

10. Gao WY, Cara A, Gallo RC, Lori F: Low levels of deoxynucleotides in peripheral blood lymphocytes: a strategy to inhibit human immunodeficiency virus type 1 replication. Proc Natl Acad Sci U S A 1993 , 90:8925-8928

11. Berger A, Sommer AF, Zwarg J, Hamdorf M, Welzel K, Esly N, Panitz S, Reuter A, Ramos I, Jatiani A, et al: SAMHD1-deficient CD14+ cells from individuals with Aicardi-Goutieres syndrome are highly susceptible to HIV-1 infection. PLoS pathogens 2011, 7:e1002425

12. Hrecka K, Hao C, Gierszewska M, Swanson SK, Kesik-Brodacka M, Srivastava S, Florens L, Washburn MP, Skowronski J: Vpx relieves inhibition of HIV-1 infection of macrophages mediated by the SAMHD1 protein. Nature 2011, 474:658-661.

13. Laguette N, Sobhian B, Casartelli N, Ringeard M, Chable-Bessia C, Segeral E, Yatim A, Emiliani S, Schwartz O, Benkirane M: SAMHD1 is the dendriticand myeloid-cell-specific HIV-1 restriction factor counteracted by Vpx. Nature 2011, 474:654-657.

14. Goldstone DC, Ennis-Adeniran V, Hedden JJ, Groom HC, Rice Gl, Christodoulou E, Walker PA, Kelly G, Haire LF, Yap MW, et al: HIV-1 restriction factor SAMHD1 is a deoxynucleoside triphosphate triphosphohydrolase. Nature 2011, 480:379-382

15. Lahouassa H, Daddacha W, Hofmann H, Ayinde D, Logue EC, Dragin L, Bloch N, Maudet C, Bertrand M, Gramberg T, et al: SAMHD1 restricts the replication of human immunodeficiency virus type 1 by depleting the intracellular pool of deoxynucleoside triphosphates. Nat Immunol 2012, 13:223-228.

16. Powell RD, Holland PJ, Hollis T, Perrino FW: Aicardi-Goutieres syndrome gene and HIV-1 restriction factor SAMHD1 is a dGTP-regulated deoxynucleotide triphosphohydrolase. J Biol Chem 2011, 286:43596-43600

17. Kim B, Nguyen LA, Daddacha W, Hollenbaugh JA: Tight Interplay among SAMHD1 Protein Level, Cellular dNTP Levels, and HIV-1 Proviral DNA Synthesis Kinetics in Human Primary Monocyte-derived Macrophages. J Biol Chem 2012, 287:21570-21574.

18. Yusuf I, Fruman DA: Regulation of quiescence in lymphocytes. Trends Immunol 2003, 24:380-386.

19. Brandariz-Nunez A, Valle-Casuso JC, White TE, Laguette N, Benkirane M Brojatsch J, Diaz-Griffero F: Role of SAMHD1 nuclear localization in restriction of HIV-1 and SIVmac. Retrovirology 2012, 9:49.

20. Laquette N, Rahm N, Sobhian B, Chable-Bessia C, Munch J, Snoeck J, Saute $D$, Switzer WM, Heneine W, Kirchhoff F, et al: Evolutionary and functional analyses of the interaction between the myeloid restriction factor SAMHD1 and the lentiviral Vpx protein. Cell Host Microbe 2012 11:205-217.

21. Siliciano RF, Greene WC: HIV Latency. Cold Spring Harbor Med 2011 1:a007096.

22. Trono D, Van Lint C, Rouzioux C, Verdin E, Barre-Sinoussi F, Chun TW, Chomont N: HIV persistence and the prospect of long-term drug-free remissions for HIV-infected individuals. Science 2010, 329:174-180.

23. de Sio FR S, Trono D: APOBEC3G-depleted resting CD4+ T cells remain refractory to HIV1 infection. PloS one 2009, 4:6571.

24. Rice GI, Bond J, Asipu A, Brunette RL, Manfield IW, Carr IM, Fuller JC, Jackson RM, Lamb T, Briggs TA, et al: Mutations involved in Aicardi-Goutieres syndrome implicate SAMHD1 as regulator of the innate immune response. Nat Genet 2009, 41:829-832.

25. Doitsh G, Cavrois M, Lassen KG, Zepeda O, Yang Z, Santiago ML, Hebbeler AM, Greene WC: Abortive HIV infection mediates CD4 T cell depletion and inflammation in human lymphoid tissue. Cell 2010, 143:789-801.

26. Zhou Y, Shen L, Yang HC, Siliciano RF: Preferential cytolysis of peripheral memory CD4+ T cells by in vitro X4-tropic human immunodeficiency virus type 1 infection before the completion of reverse transcription. J Virol 2008, 82:9154-9163.

27. Cameron PU, Saleh S, Sallmann G, Solomon A, Wightman F, Evans VA Boucher G, Haddad EK, Sekaly RP, Harman AN, et al: Establishment of HIV-1 
latency in resting CD4+ T cells depends on chemokine-induced changes in the actin cytoskeleton. Proc Natl Acad Sci U S A 2010, 107:16934-16939.

28. Welbourn S, Miyagi E, White TE, Diaz-Griffero F, Strebel K: Retrovirology 2012, 9:86 (23 October 2012).

29. Goncalves A, Karayel E, Rice Gl, Bennett KL, Crow YJ, Superti-Furga G, Burckstummer T: SAMHD1 is a nucleic-acid binding protein that is mislocalized due to aicardi-goutieres syndrome-associated mutations. Hum Mutat 2012, 33:1116-1122.

doi:10.1186/1742-4690-9-87

Cite this article as: Descours et al:: SAMHD1 restricts HIV-1 reverse transcription in quiescent CD4 ${ }^{+}$T-cells. Retrovirology 2012 9:87.

\section{Submit your next manuscript to BioMed Central and take full advantage of:}

- Convenient online submission

- Thorough peer review

- No space constraints or color figure charges

- Immediate publication on acceptance

- Inclusion in PubMed, CAS, Scopus and Google Scholar

- Research which is freely available for redistribution 